\title{
RULE-BASE GENERALIZATION METHOD ON LARGE-SCALE TOPOGRAPHIC MAP
}

\author{
XIAO Zhou ${ }^{a, b}$ Yang Bogang ${ }^{a, b}$ ZHANG Haitao ${ }^{a, b}$ \\ ${ }^{a}$ Beijing Institute of Surveying and Mapping, No.15 Yangfangdian Road,100038, Beijing China, \\ ${ }^{\mathrm{b}}$ Beijing Key Lab of Information Engineering in City Space, 100038, Beijing China \\ xiaozhou@bism.cn
}

KEY WORDS : large scale topographic map; cartographic generalization

\begin{abstract}
ABS TRACT:
Map generalization is a procedure involving much intellective reasoning action, with very wide domain. It is also a difficult problem in the field of cartography in the world. This paper makes a study on the interactive and rulebased digital generalization, and a map generalization environment for large scale topographic map is designed and realized. A number of tests have proved that map generalization can be successfully and interactively done with the cooperation of human and computer if the procedures of map generalization are wisely decomposed. Compared with the traditional manual method, this map generalization can shorten the working time to 1/4 or even. Besides, the work will become less intensive with higher precision.
\end{abstract}

\section{INTRODUCE}

Making traditional manual generalization map, it must require not only the rich-professional knowledge and work experience workers, and also having a high level of operation and skills. Normally, it is work difficulty, low efficiency, long duration, and having very large man-made error ${ }^{[1]}$.In recent years, along with the national economy developing well and fast, the level of urbanization is getting improved continuously. And the scale of urban is also becoming larger and larger. In order to facilitate plan and manage the urban area and the area which will develop urban, we need the speed of updating large scale topographical mapping products quickly. Apparently, the original manual operation mode already cannot satisfy the requirements of updating the generalization of map product, and cannot keep up with the rapid development of information technology.

So, using the new technology to realize automatic or interactive semi-automatic cartographic generalization, we can achieve the fast updating between various relevant scale map during the same region. It not only can reduce the data sources repeat purchase and updating operations investment in same region, but also offer a useful imperative method to makeup the data leaks.
Under this new digital environment for map generalization, the operator will be away from heavy and inefficient work. So using new technology to improve the efficiency of map compilation is a development direction of map drawing, which in order to adapt the new requirements of information survey ing map.

Along with the AutoCAD widely apply in industry of surveying and mapping, digital survey ing and mapping products become main means for institute of surveying and mapping. This provides convenient for automatic map generalization ${ }^{[2]}$. But the different scale topographic maps have various different requirements, such as the size of the symbol, drawing capacity, there isn't any software can realize the completely automatic map generalization. However, these years the researches for automation generalization have never stopped. Some of the current commercial software provide a number of functions, but from a practical point of view, mostly professional graphics software or module only focused on the algorithms of automatic generalization research, not for how to use the integrated algorithm effect consistent generalization.

According to the current stage of the technical condition, This paper analyzed the main factors of the influence of cartographic generalization.Through production practice, we put forward consistency cartographic generalization based on rule base and interactive graphics synthesis method. We innovate 
cartographic generalization algorithm, so form an efficient generalization process.

\section{THE S ITUATION OF LARGE SCALE TOPOGRAPHIC MAP GENERALIZATION}

Object expression entity of Large scale map is poor than small scale map, which is has complex geometry structure ${ }^{[3]}$. That's because the expression is too refined. Such as in 1: 2000 map, the house is not a simple polygon, which is affiliated with balcony, columns, stairs and other linear structure. These objects are combination, the feature representation of complex is greatly increased work intensity by a single compilation tools integrated graphics, and also makes map identification of additional difficulty.

There are some topographic map generalization software currently, which have only a few application functions through independent command in the toolbox method (such as line simplification, polygon merging ), or make these the function integrate to get more complex functions ${ }^{[4]}$. Howev4er, these features did not fully consider the different data during different coverage area which reflect the different objects. That means software designing is not flexible, not research the more suitable method for current technology and specific application examples. In this case, the process of production lack of efficient methods to improve the overall efficiency. For example, many good softwares which are good at in the ordered terrain application comparison is very difficult to play a role in unsystematic old city. So it is not difficult to find that cartographic synthesis automation doesn't lack the corresponding synthesis algorithm, but a comprehensive solution.

\section{RULE-BASED CONS IS TENCY OF CARTOGRAPHIC}

Through the production practice and understanding the generalization rule, we found a rule base to management and implementation. The rule base is one or more integrated scheme of generalization mathematical model which has regional or characteristic index. Construction of rule base can be achieved by experience graphic-product staff, including cartographic generalization rules and parameters, flow, and then other common operating staff product in accordance with this comprehensive rule base. Thus, rule base can compensate the different understanding of standard specification for every worker, and different drawing experience, aesthetic differences, as well as different specific object processing method, which may result defects of effect differences form respective map generalization. This rule base provides improved quality and consistency of effect control mechanism for the process. And implementation and application of rule base are important work foundations for fully automated and intelligent generalization.

The mathematical model form rule base is defined basic operator components which are related cartographic generalization, such as merging operator, simplification operator, distance operator, neighborhood search operator. At the same time, model open up setting interface of generalization parameter. Setting different parameters of the model, you can realize different comprehensive effect, such as the poly gon merge tool base on Delaunay. The rules are combination with parameterized model and logical, rule base is the combination with rules.

In general, we can identify the specific parameters when we want to use the specific rules. The instantiation executable program for rule base comprehensive on the specified data automatically. Firstly, we put the thematic elements cartographic generalization method and parameter as a rule in the rule base, which will form a complete set of drawing comprehensive scheme. Rule designer and program designer are used to generate rules files and program files. Rules file can be seen as a series of rules collection. Because the rules have not been instantiated yet (not specified processing elements and layers), we can't directly integrate through the rules to cartographic generalization. Program is the rule collection which has been instantiated. Through the program file, we can achieve to comprehensive the specified data automatically. The Figure 1 shows the relationship between the rules, rules base, program designer and cartographic software.

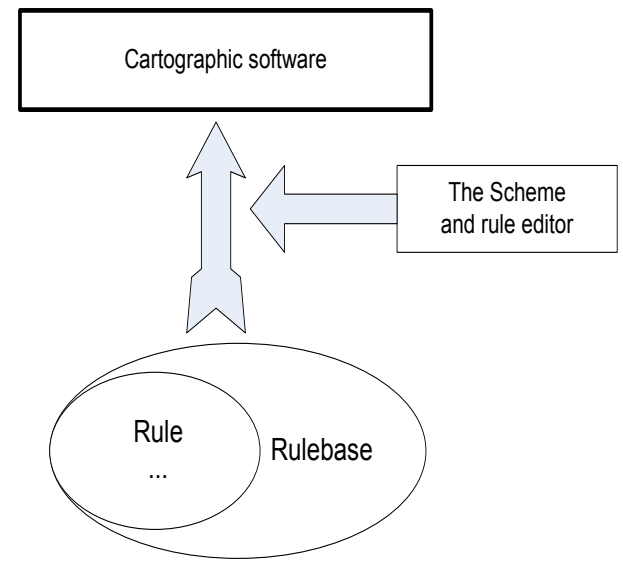

Figure 1 The relationship between the rules, rules base, rule editor and cartographic software 
This would solve the disadvantage that common software cannot adapt to the different topographic features elements in processing. At the same time, the most comprehensive process can be executed directly in the rule base. And operator doesn't need to find the menu, toolbar, which avoids the mouse moving frequently caused wasting time.

In order to verify this method, we did comprehensive practice work in Beijing area. First, technical experts who have wealthy experience of generalization complete the development of the rule base and implementation plan. Their rich experience becomes suitable data operations drawing comprehensive program file through the rule designer and project designer, and then distributed to the common operator. The operators follow the program file, in accordance with selected rules and parameter, thus complete the expert level of cartographic production.

However, the rule base batch synthesis cannot reach $100 \%$ full automatic integrated, so the current system of batch processing integrated positioned to solve those high repeated operations relatively, and comprehensive is not too complicated. These will emancipate the workers from heavy repetition operation, so greatly improve the production efficiency.

\section{INTERACTIVE COMPREHENSIVE GENERALIZATION}

Automatic map generalization is a highly complex and intelligent process, there is not a software can realize the completely automatic map generalization in China. From the features of large scale topographic map we can see these features are more detail and the relationship is more complicated. Nowadays the automatic synthesis of basic theory still immature condition, it's not realistic to achieve the fully automatic generalization in such complex information relationship.

So one reasonable solution is using both the interactive and rulebased digital generalization. Interactive comprehensive is mainly through a variety of comprehensive tool for man-machine interactive way to semi-automatic integrated processing. And also can use the previously mentioned in rulebased model. The complicate problem is split into a number of small problems. The parts which can solve automatically we use the software automatical function as far as possible, and the parts which cannot be solve automatically we add a few manual interventions to artificial interaction. We can also customize with rich interactive automatic synthesis tool, and through the overall user experience and tools to achieve flexible satisfactory combination generalization effect. A variety of means are used to improve the level combination of comprehensive automation, thus achieve the goal of improving production efficiency.

\section{ARCHITECTURE OF S OFTWARE S YS TEM}

In this paper, we maked the Beijing 1:2000 to 1:10000 generalization topographic map using integrated system as example, put forward a rule-based interactive generalization integrated software system. In this cartographic generalization system we have three levels or ways of generalization, automatical digital rulebased generalization, interactive and comprehensive manual editing.

The software system is divided into four layers: data layer, core layer, logic layer and business layer.

The data layer is composed by the original" large scale terrain data"," comprehensive generalization of historical action data" and" new small scale terrain data", they constitute the comprehensive project. The "comprehensive generalization of historical action data " is the process record in cartographic generalization keeping systems do the various actions, which is in order to facilitate the comprehensive action replays, and also for the subsequent data updated automatically to provide reference.

The core layer includes the data model and the correlation function and the algorithm of data processing, and provides support for the logical layer.

Logic layer, in based on the kernel, using a standardized interface, developed three relative independent component bases: cartographic generalization rule processing component base, automatic integrated operator component base and feature integrated component base. Cartographic generalization rule processing component base is based on the implementation of the rules of automatic synthesis process. Automatic integrated operator components base provides the basis and the general automatic integrated processing algorithms (such as selection, simplification, and etc.). Thematic integrated component base in automatically synthesizing operator base foundation, achieved for the road, buildings, water, landforms, vegetation and other complex feature custom integrated; logic layer using components to realize the idea, help reduce the module coupling level, improve stability, independence and openness of the logical layer.

In the business layer, integrated graphics software is divided into 7 modules: the data exchange module, 
integrated processing module, processing module, subject to rules for processing module, interactive editing module, quality checking module and system management module. See figure 2 :
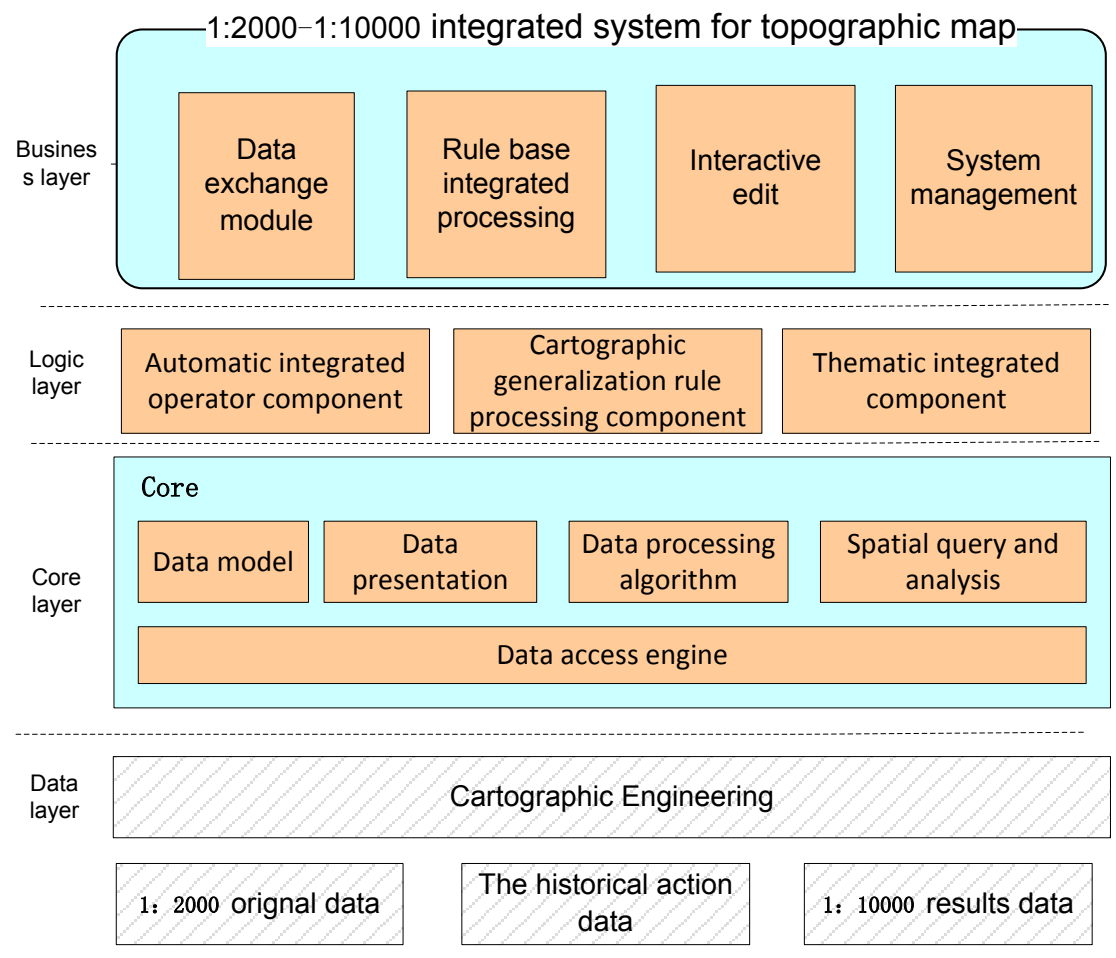

Figure 2 The integrated system for topographic map 


\section{APPLICATION}

The author used Beijing 1:500, 1:2000 and 1:10000 topographic map data as an example for generalization production experiment. In the generalization of the experimental work, first, we combined with the related mathematics model and method, found the Beijing topography data characteristics, due to the map generalization specifications, designed drawing comprehensive quantitative index, which is as every element selection reference and control parameters. The terrain is divided into nine layers. each layer object should be integrated according to the order of execution. At the same time, we combined the features of attribute information and the related mathematical models were judged.

\subsection{The comprehensive function for Residents}

The main operations for residents integrated are simplification, neighbouring polygon merging and resident level transform. According to the requirements of comprehensive, this can be used to automatically merge though operator representation. Various parameters were recorded in the rule base, we can use interactive tools for generalization operation. Figure 3 shows that the differences before and after comprehensive. In the process of integrated, urban and suburban residents were used in different ways. Group of urban residents used combined operator, which correct the implementation of building corner vertical characteristic. Suburban parts did not use this operator, and should be alone with the characteristics of linear features ( such as railway, river ) or the trend of contour, and keep the whole range of the residents. For relatively simple polygon features, we can design level of transformation in the rule base ,and then do the batch processing.

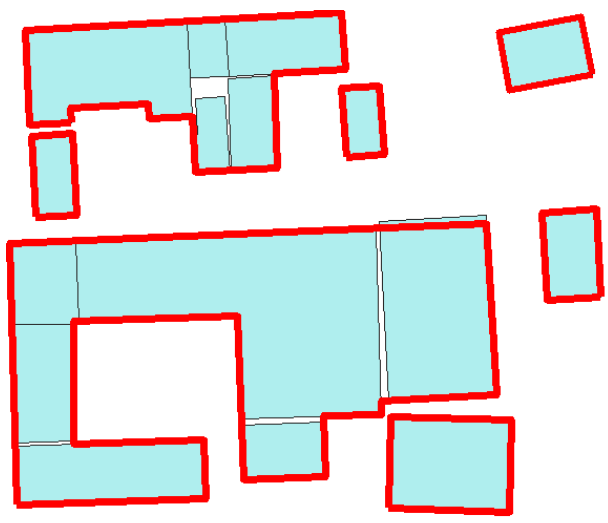

Figure 3 The Residents comprehensive

\subsection{The comprehensive function for River}

Comprehensive factors of river are including river selection, simplification, merging of adjacent lakes, narrow river operation for axis. When the width of river is less than the parameter which has been set in rule base, the axis extraction operator will integrate to single line river automatically. After selecting single line river, we need to streamline simplification, make the curved river smooth. These operate can be used in batch mode to handle rule base.

\subsection{The comprehensive function for Road}

After the scale cartographic generalization, original roads became dense and narrow. So that, comprehensive selection functions of road are the main operations. In accordance with the level, length and the relationship of road, we did condition selection. Such as, the highway, road, substandard-highway have been directly reserve (batch action by rule base), and then use interactive tools to realize road centerline extraction process. See figure 4.

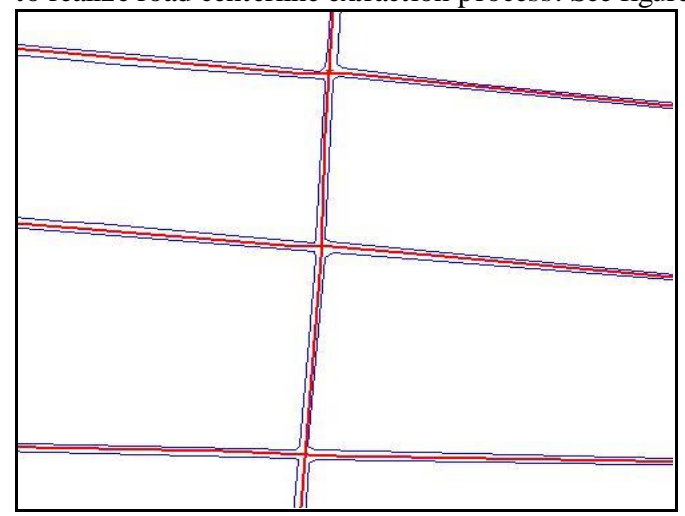

Figure 4 The Road comprehensive

\subsection{The comprehensive function for Terrain}

The contour of terrain is the most commonly used method, and also the main content of landscape, which needs to sift and simplify during generalization.

We use the automatic processing functions of rule base for terrain contour processing, set the contour interval which is preserved and the parameters of minimum simplification, then do the sift and simplification for terrain contour.

There are two steps for generalization elevation points. One is setting the feature elevation points firstly by manual selection, such as points on road, bridge, at the top of the mountain etc.; secondly, it's need to consider the evenly distributed of points, and annotation does not cover features and other factors, finally do automatic extracting for other elevation points. See figure 5 . 


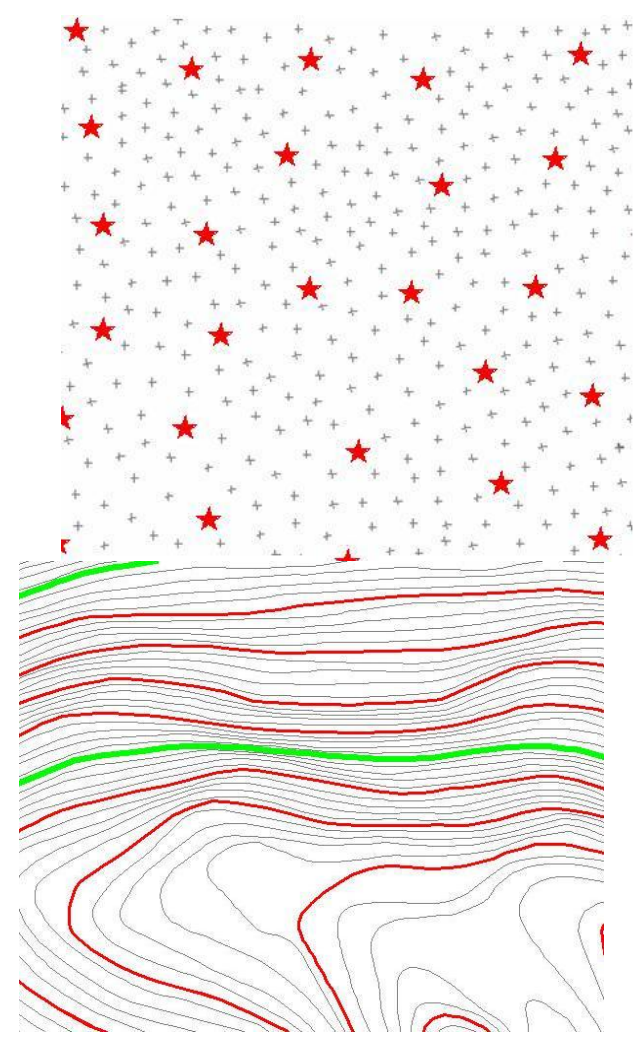

Figure 5 The terrain comprehensive

\section{CONCLUSION}

Through the practical application, it proves that the rule-based consistency of cartographic generalization and interactive graphics synthesis method of combining operation have relatively high efficiency. And based on this interactive environment, it can make the operator in the digital environment to do the generalization. From the actual effect, comprehensive result basically accord to the requirement of generalization, it is reasonable and effective to do the whole process of generalization operation. From the work intensity, this method improves the original software and processing based on AutoCAD, makes the graphics data relative achieve more automatically cartographic generalization. It can be gradually adapt to the keeping increasing he amount of generalization task in the future. In addition, from the work time cycle, it can be completed in shorter $1 / 3$ or less time than the original. And the operation accuracy is also greatly improved, the comprehensive data can be directly put in Database. In this case, it can greatly reduce the personnel cost, shorten the cycle, improve data quality and processing efficiency.

\section{Reference}

FU Zi-ao,ZHU Jiang,CAI Jinsong. 2002 Research on Symbol and Code of Large Scale Topographic Maps[J].ACTA GEODAETICA ET CARTOGRAPHICA SINICA 2002, 02(37):37-41
CHAO Chun-hao,ZENG jingwen. 2004 Application Studying on Topographic Map Symbols List of Beijing[J].Beijing Surveying and Mapping. 2004, 42(3):10-15

LIU Yaolin\&Martin Molenaa .2003 Categorical Database Generalization[J].Geo-spatial Information.2003 6(4):19-24

CAI Zhongliang, Wu Hehai,DU Qingyun,LIAO Chujiang,2003,Interactive Generalization on Large-Scale Topographical Map Supported by a Datebase Platform[J].Geospatial Information.2003 6(4):17-26

WEI Xudong, 2006,Research on Digitizing Method of Medium Scale Topographic Map [J].GeoMatics\& Spatial information technology.2006 29(1):57-63 